\title{
Proceedings of the International Workshop "Shapes and Dynamics of Atomic Nuclei: Contemporary Aspects" (SDANCA-21), 16-18 September 2021, Sofia, Bulgaria. PART II
}

\author{
doi: https://doi.org/10.55318/bgjp.2022.49.1.001
}

This Volume contains the second part of articles based on presentations given at the International Workshop "Shapes and Dynamics of Atomic Nuclei: Contemporary Aspects" (SDANCA-21) in continuation of the contributions published in Vol. 48 No. 5-6 (2021) of this journal. The Workshop SDANCA-21 was held in Sofia Tech Park, Sofia, Bulgaria from the 16-th to the 18-th of September 2021. It was organized by researchers from the Institute of Nuclear Research and Nuclear Energy (INRNE) in Bulgarian Academy of Sciences (BAS) with the financial support of the Bulgarian National Science Fund under contracts No. KP-06-N48/1, KP-06-N28/6 and KP-06-RILA/6. This is the fourth issue of the workshop SDANCA-15, SDANCA-17 and SDANCA-19 held in Sofia in 2015, 2017 and 2019.

The aim of the Workshop was to gather researchers in the field of nuclear shape dynamics and related areas providing a possibility to discuss advanced ideas, actual problems and future developments in the subject. The meeting outlined several directions of common interest and inspired new ideas in various contemporary aspects of the theoretical and experimental approaches to the shapedynamics problem in the nucleus and other physical systems. We believe that it contributed to the strengthening of the existing collaborations and inspired new activities on the topic.

The SDANCA-21 Workshop covered the following main topics:

- Collective and microscopic theories of nuclear deformation;

- Shapes and symmetries - geometric and algebraic aspects;

- Shape coexistence and shape-phase transition phenomena;

- Complex shapes, isomers and cluster phenomena;

- Shapes and dynamics at the limits of stability;

- New data on nuclear deformations and current experimental techniques;

- Interdisciplinary aspects: links to shape phenomena in other physical systems.

The Workshop was attended by 68 participants (30 onsite and 38 online) from 18 countries: Czech Republic, Denmark, France, Germany, Greece, Hungary, 
India, Israel, Japan, Morrocco, Romania, Russia, South Africa, Switzerland (CERN), Turkey, United Kingdom, USA and Bulgaria.

The scientific program contained 54 talks in total ( 23 onsite and 31 online) covering issues of microscopic and collective approaches and new experimental results as well, including: density and covariant-density functional theory, manybody/self-consistent theories, non-core shell and ab-initio developments, grouptheoretical (algebraic) models exploring different kinds of symmetries (unitary, symplectic, "proxy", "pseudo", "partial dynamical", etc.) associated with the most actual effects of shape coexistence, shape-phase transitions, shell effects, pairing and multipole interactions, geometric models treating rigidity and softness, triaxiality and chirality, pear-shape and clustering effects, nuclear masses and moments, high- $K$ and "clock" isomers, properties at different mass regions ( $A=130,180 ; N=90,116-122 ; N \sim Z$, superheavy, etc.). Current developments of experimental techniques, facilities and collaborations, such as ELINP, TRIUMF-TITAN, FRS-IC, COLLAPS/ISOLDE, INGA and others, were presented together with new data from lifetimes, moments and mass measurements and analysis. Latest achievements in interdisciplinary subjects such as RHIC/CGP, neutron stars, pionic helium, lasers and nuclear clock elaboration were discussed in the aspects of their relation to atomic nuclei. A commemorative discussion devoted to the late Prof. David Rowe was held and a tribute paper is now published in this Volume, giving respect to his pioneering works in the group-theoretical approach and their impact on the new generations of researchers in the field. During the preparation of the Volume we learned that in December 2021 his close collaborator, long-years co-author and also prominent expert in the field, Prof. George Rosensteel passed away. Here we express our deep respect to these two great scientists.

Further details about the SDANCA-21 workshop can be found in the Workshop web site: http://ntl.inrne.bas.bg/events/sdanca21/.

This Volume includes 10 articles completing together with Vol. 48 No. 5-6 (2021) a collection of 39 contributions to the Workshop Proceedings.

I would like to express my thanks to all participants of the SDANCA-21 Workshop for their contribution to the inspiring scientific atmosphere, to all authors who contributed to both Volumes, as well as to all referees who helped with their highly expert comments for the high quality of the included articles.

January 2022, Nikolay Minkov 


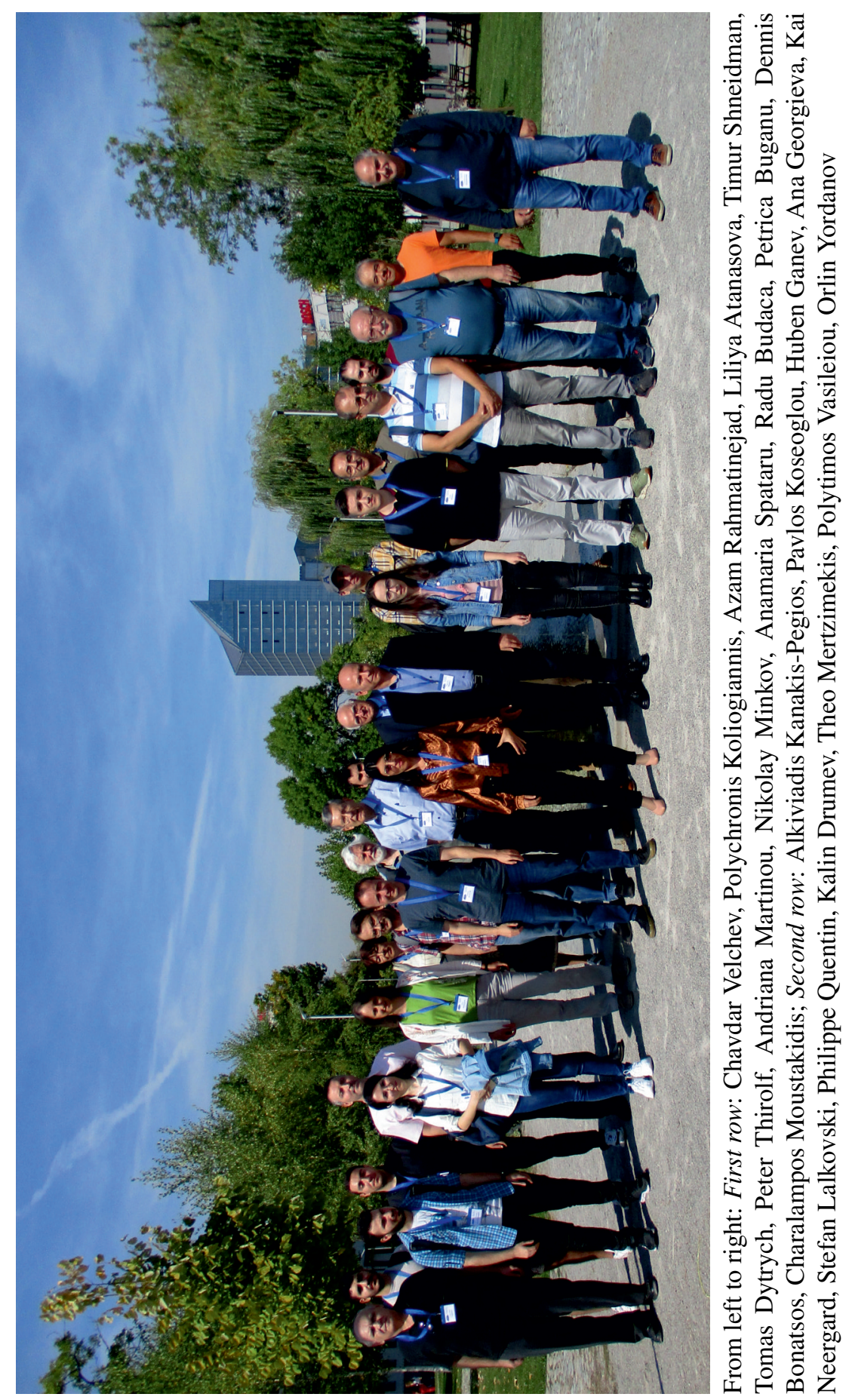




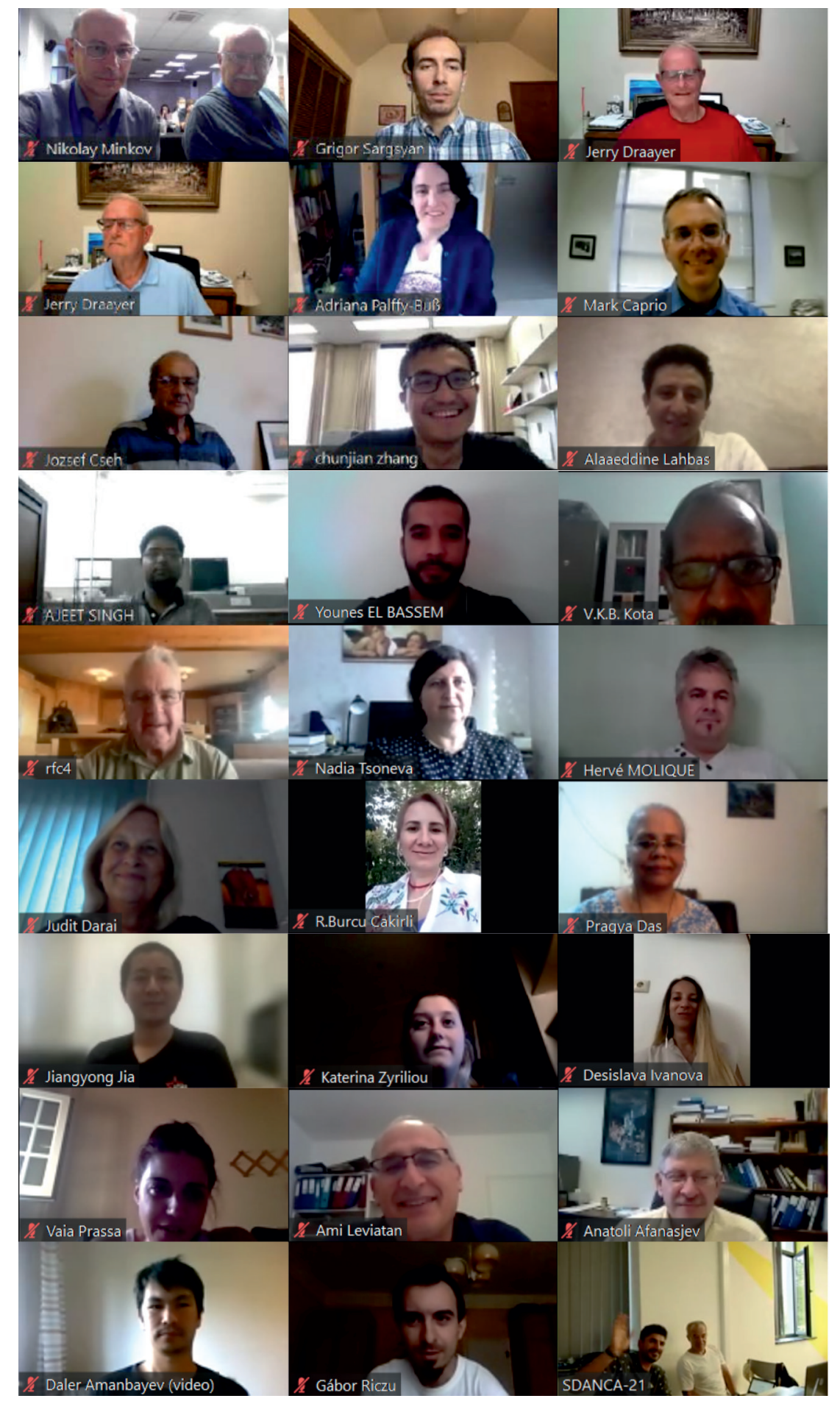

